\section{DIRECT CHEMICAL ACTION UPON NERVE CENTRES IN BIOLOGY AND MEDICINE}

\author{
By PROF. LENA STERN \\ Physiological Institute, Academy of Sciences of \\ the U.S.S.R., Moscow
}

$\mathrm{I}_{\mathrm{r}}^{\mathrm{N}}$ the course of evolution, as organisms have become more complex, they have also become more sensitive to their internal environment, for example, in higher animals, to the temperature, $p \mathrm{H}$, and osmotic pressure of the blood and lymph. Minute changes in the properties of the internal milieu may result in serious dislocation of the functioning of the organism. As Claude Bernard said: "La fixité du milieu intérieur est la condition de la vie libre".

\section{Local Environment}

In addition to the general internal milieu common to all parts of an organism, there has evolved for each individúal organ a specialized 'local' milieu, for which we have proposed the name 'immediate' or 'intimate' environment. Each organ derives the materials necessary for its functioning from the cellular fluid forming its local environment; and into this local environment the organism discharges the products of its metabolism. The physical and chemical properties of the fluid which is the local environ. ment of an organ are related to the structure and functions of that organ. Any abnormal change in the composition of this environment leads to a corresponding disturbance in the organ; the disturbance may be so serious as to bring about the death of the whole organism.

The local environment of each organ is separated from the general internal environment by a physiological mechanism to which we have given the name 'histo-hæmatic' barrier. These barriers regulate the exchange between the blood and the immediate environment of each organ ; they control the inward passage of substances from the blood stream; they facilitate the discharge from the local environment of the organ into the blood stream of the products of metabolism ; they maintain the constancy of the local environment.

It is clear, therefore, that the proper functioning of each organ depends on the integrity of the histohæmatic barrier surrounding its local environment. For example, an increase in the permeability of the barrier would expose the organ to any drugs circulating in the blood, which might not be able to penetrate the barrier under normal conditions.

\section{The Hæmato-Encephalic Barrier}

The spinal fluid is the local environment of the brain and the spinal cord. We have found that the properties of the spinal fluid depend largely upon the regulating action of what we call the 'hæmatoencephalic' barrier, which comprises the endothelium of the capillaries and the neuroglia. This barrier protects the central nervous system from toxins, virus, bacteria, etc., in the blood stream ; it controls the entry of substances necessary for the nutrition of the central nervous system; it allows the passage from the spinal fluid into the blood stream of metabolic products of the brain which would, if they accumulated, disturb the activity of the central nervous system. The hæmato-encephalic barrier possesses selective permeability not only for substances foreign to the organism, but also for various natural products of metabolism, for example, antitoxins, hormones, etc. The permeability is, moreover, asymmetric: there is more selection in the direction blood $\rightarrow$ spinal fluid than in the direction spinal fluid $\rightarrow$ blood. This concept of histo-hæmatic barriers (particularly the hæmato-encephalic barrier) has been the basis of our work on the differences between the effects of drugs injected into the blood stream and into the fluid of the spinal cord.

There is a close relation between the permeability of the hæmato-encephalic barrier for any particular substance in the blood, and the possibility of this substance affecting the central nervous system. The only substances which affect the central nervous system when added to the blood are those which penetrate into the nutritional milieu of the brain, that is, the spinal fluid. Other substances which normally produce no neurotropic effect may affect the nervous system profoundly if they enter the spinal fluid. Consequently, when defining the neurotropic properties of a substance, it is necessary to know whether or not the substance has penetrated the hæmatoencephalic barrier, and been admitted to the immediate environment of the nervous system.

It is of considerable theoretical and practical interest that the selective permeability of the hrmatoencephalic barrier is considerably less in new-born animals with an undeveloped brain (for example, dog, rabbit, cat, rat) than in adult animals of the same species. This is probably true, too, for the newborn human being. This view is consistent with two facts: it is known that the substances introduced for therapeutic purposes into the circulatory system of a new-born child have effects quite different from the same substances in an adult; and the peculiar clinical manifestations of some diseases in the child (cramps, high temperature) are consistent with the view that in the child toxic substances present in the blood pass into the spinal fluid much more easily than they do in an adult. It is our view that there is a parallel development of the central nervous system and the hæmato-encephalic barrier, not only ontogenetically but also phylogenetically.

The defensive action of the hæmato-encephalic barrier not only increases during early development; it also varies according to the physiological condition of the organism. In pregnant animals, for example, the permeability of the barrier is easily changed by chemical or physical agents which scarcely affect the barrier in animals which are not pregnant. Animals heavily drugged with alcohol show, in the same way, a much greater sensitivity of the hrmato-encephalic barrier: in such animals even a slight change of temperature affects the permeability of the barrier.

The selective permeability of the hæmato-encephalic barrier safeguards in health the nerve centres against invasion by toxic substances. But in sickness, the barrier may be a hindrance, because it may prevent the penetration of therapeutic substances to the diseased nerve centres. This view is supported by clinical data. We have evidence that the pathological symptoms of the nervous system in some infectious diseases (for example, tetanus, encephalitis) are due to the direct action of the toxin or virus concerned upon the brain. We have evidence, too, that attempts to cure disorders of the central nervous system by the application of therapeutic agents into the blood stream are ineffectual because the barrier prevents the agents from passing into the spinal fluid. 


\section{Direct Introduction of Substances into the Spinal Fluid}

There are two ways in which a therapeutic agent can be brought into the local environment of the central nervous system : (1) by lowering the resistance of the barrier (by increasing its permeability) and (2) by avoiding the barrier (by introducing the substance directly into the cavity of the cerebral ventricles).

It is possible through changes of temperature, $p \mathrm{H}$, osmotic pressure, etc., to increase the permeability of the hremato-encephalic barrier. But the clinical value of this treatment is questionable, for the permeability is increased not only to the substance it is required to introduce, but also to any noxious substances and toxins which may be in the blood stream. The second method, of introducing the substance directly into the cavities of the cerebral ventricles, has been intensively studied by us in recent years. We have, for example, introduced antitetanus serum into animals suffering from tetanus. In all experiments where the serum was introduced into the circulation, it gave positive results if introduced before the first symptoms of tetanus appeared. In experiments where the serum was introduced into the cerebral ventricles even after the onset of tetanus, the disease was arrested and there was total recovery : provided, of course, that the interval between the onset of symptoms and the time of injection was not too great.

These results secured with experimental animals have been applied successfully to horses suffering from tetanus and have been confirmed by clinical practice. Patients suffering from tetanus have been injected with anti-tetanus serum by cisternal injection. In the great majority of cases, if the injections were applied in time, the patients improved rapidly. This treatment is now being applied successfully in Army hospitals : simultaneously with an intravenous or intramuscular injection, $10-20 \mathrm{ml}$. antitetanus serum-that is, 15,000-30,000 American units-are introduced by cisternal injection into the cerebro-spinal canal, after removing an equäl volume of spinal fluid. The method has recently been applied by Prof. E. M. Steblov, of the Neurological Institute in Alma-Ata, to treat the after-effects of seasonal encephalitis. Twenty-two cases were treated with reconvalescent serum. In only two of these cases, where the syndrome was of more than twenty years standing, did the injections produce no effect. In the other twenty cases there was visible improve. ment after the fifth injection, and in some patients even some power of movement was restored.

It is possible that the method may be extended to treat successfully other disorders of the brain and nervous system, even by the introduction direct into the spinal fluid of the patient's own blood serum.

\section{Antagonistic Action of Chemicals}

The work of $O$. Loewi and others has shown that nerve action is aecompanied by the production of mediators: chemical substances resembling adrenaline and acetylcholine. These mediators have the same sort of action on effectors as direct stimulation by a corresponding nerve; they support and enhance the effects of excitation on the peripheral parts of the vegetative nervous system.

Our experimental results, and others already published, demonstrate that these mediators have a precisely opposite action on the corresponding parts of the central nervous system. They penetrate the hæmato-encephalic barrier from the blood stream to the spinal fluid, and there they tend to neutralize the effect they have produced on the peripheral system. These mediators, by neutralizing at on $\theta$ pole of the nervous system the effects they have produced at the other pole, provide a means of autoregulation in the organism. Their antagonistic effect is shared by humeral agents, too.

The work of a great number of scientific workers indicates that the metabolites of the brain exert a similar autoregulating function. Substances produced in the central nervous system (for example, substances present in blood flowing from the brain) affect the peripheral elements quite differently from the central nervous system. The conclusion can be drawn that these chemical substances, by their contrasting action on different parts of the organism, play an important part in autoregulation. The brain controls not only by nervous impulses, but also by liberating chemical agents.

Arising out of this antagonism, there is another application of the method of direct injection into the spinal fluid, which depends on the fact established by us, that the effect of some inorganic substances (for example, salts of potassium and calcium) on the peripheral elements of the vegetative system and their effector organs differs totally from the effect of the same substances on the central nervous system. Thus it has been established that an increase in the quotient $\mathrm{K} / \mathrm{Ca}$ in the spinal fluid increases the tonus of the sympathetic nervous system; but in the blood the same changes in the quotient $\mathrm{K} / \mathrm{Ca}$ lead to diametrically opposite results. It is sufficient to introduce a minimal quantity ( $\mathrm{a}$ few mom.) of $\mathbf{K}$ ions into the cerebral ventricles to call forth a strong excitation of vegetative organs ; augmentation of cardiac activity; increase of muscle tonus, metabolism and excitability : all results diametrically the opposite of those produced if potassium is introduced into the blood stream. The same contrast is brought about by certain other materials (metabolites of organs, hormones, mediators, etc.) when applied to the blood on one hand and to the spinal fluid on the other.

\section{Treatment of Shock}

Since many pathological states, including vegetative neuroses, are due to changes in the tonus of the sympathetic and parasympathetic centres, or in the relation between the tonus of these centres, the method of direct application of drugs to these centres has considerable clinical possibilities. The results we have obtained in the treatment of shock in the wounded are of particular interest.

The role of the vegetative nervous system in the development of shock has been developed especially by W. Cannon and his collaborators, and it has been confirmed by many other workers and by ourselves. Shock is directly due to changes in the tonus of the nervous system, in particular to the diminution of the tonus of the sympathetic centres following their extreme excitation. An analysis of the spinal fluid in the later stages of shock shows a decrease in the quotient $\mathrm{K} / \mathrm{Ca}$, owing mainly to a decrease in potassium. This is accompanied by a spontaneous decrease in inorganic phosphate. These observations lead us to the conclusion that the first task in the treatment of shock is to introduce potassium phosphate directly into the cerebral ventricles in order to restore the $\mathrm{K} / \mathrm{Ca}$ quotient. 
This treatment proved effective on animals. Soon after the introduction of the solution, blood pressure increases rapidly, respiration becomes deeper, the excitability and reactivity of the animal are restored. The injection must be performed as early as possible because the capacity of the vegetative centres to respond to treatment decreases during shock. Clinical treatment of shock confirms these experiments. The method now being successfully applied to war cases is to introduce suboccipitally to the vegetative centres a mixture of mono- and di-potassium phosphates $(6.5 \mathrm{mgm}$. $\mathrm{K}$ per ml., with a $p \mathrm{H}$ of $7 \cdot 4-7 \cdot 5)$. For clinical treatment the quantity used ranges from 2 to $7 \mathrm{ml}$., according to the severity of the shock, and for animals, $0 \cdot 1 \mathrm{ml}$. per $\mathrm{kgm}$. live-weight. The patient reacts in one or two minutes. It is essential that the material should penetrate the ventricle cavity; failure of the treatment is usually due to technical errors in its administration.

\section{Other Possible Applications}

The results encourage us to suppose that the method of direct action upon the nerve centres may be effective in the treatment of other nervous disorders. For example, diseases believed to be due to excessive tonus of the parasympathetic system (vagotonia) or of the sympathetic nervous system (sympatheticotonia) might be treated by introducing into the cerebral ventricles potassium phosphate in one case and calcium salts in the other. Promising results have been obtained by Prof. Y. Y. Sendulsky of Alma-Ata, in the treatment of certain kinds of deafness associated with the vegetative nervous system by cisternal injections of potassium phosphate, and for some kinds of dystrophia and chronic sepsis, by injection of vitamin $B_{1}$.

A further extension of our investigations in recent years has revealed that certain metabolites of organs (for example, the thyroid gland) affect the nervous system in a way quite different from their effects through the blood stream. There is an interesting field for the study of the effects of other metabolites on the nervous system. It is possible that, by the use of this method of direct injection into the spinal fluid, some diseases of the nervous system hitherto regarded as incurable may yield to treatment.

\section{PHYSICAL AND CHEMICAL DEGRADATION OF RUBBER}

A

THOUGH chemical unsaturation is not essential to the occurrence of 'rubbery properties', all forms of 'rubber' which to-day find extensive application consist of long-chain molecules with frequently recurring ethylenic linkages. Admittedly, the chemical unsaturation may, and does, have adverse influence on the service life of rubber articles, but it also enables the material to be submitted to various forms of chemical treatment for the fuller control of its manipulation during manufacture and for enhancement of the physical characteristics of the manufactured products. Fortunately, the degree of chemical reactivity is so moderate as to permit a practical compromise leaving a large credit balance on the side of mechanical stability and endurance.

It is difficult to dissociate sharply the phenomena associated respectively with the chemical and physical instability of rubber. They have been of very great importance ever since the inception of the rubber industry. The deterioration of some of his early products was a grievous trouble to Charles Goodyear a century ago, and would have daunted a less pertinacious and resilient pioneer. Thanks to his basic achievements in the United States and to those of Thomas Hancock in Britain, supplemented by other discoveries, the rubber industry has made striking advances, and rubber articles are to-day capable of long service under conditions which then would have been deemed quite impracticable.

The recent achievement of the American chemical industries in producing synthetic rubber on a scale commensurate with the pre-war output of the rubberplantation areas has incidentally necessitated still greater attention to the deliberate and controlled 'breakdown' of rubber; even more than natural rubber, the chief new material, so-called GR-S synthetic rubber, demands plasticization as a preliminary stage in its conversion into manufactured products.

In view of the many-sided importance of the subject and its associated problems, a symposium was held in Birmingham on May 12, on the initiative of the Midland Section of the Institution of the Rubber Industry, to discuss the "Physical and Chemical Breakdown of Rubber". As stated at the symposium by its chairman, Mr. F. G. W. King, technical director of the Dunlop Rubber Co., the magnitude of the contribution of the rubber industry to the allied war effort is difficult to appreciate fully, and the diffculties which have been surmounted are realized only by those directly concerned. Many of these difficulties fall within the range of the subject discussed.

The nine papers submitted revealed the breadth of the research activities of the industry, three contributions being presented by the British Rubber Producers' Research Association, two by the Research Association of British Rubber Manufacturers, two by the Rubber Division of Imperial Chemical Industries, Ltd., and two by the Technical Department of the Dunlop Rubber Co., Ltd.

The first paper to be read gave a preliminary report by Drs. W. J. C. Orr and J. L. Bolland, of the British Rubber Producers' Research Association, on the "Thermal Breakdown of Rubber" observed with comminuted 'sol' rubber, carefully freed from dissolved oxygen. Up to $200^{\circ} \mathrm{C}$., the rubber is stable; but at $220-270^{\circ} \mathrm{C}$. it shows gradual degradation without substantial loss of unsaturation but with the formation of a small proportion of volatile products, mainly isoprene and dipentene, approximately one molecule of the former and four of the latter being found when the molecular weight of the rubber, by osmotic measurements, has fallen to half its original value. On further heating, the rubber again becomes more viscous and finally sets to a benzene-insoluble, non-sticky solid. This latter change indicates that the degradation mechanism must be complicated by secondary reactions between the intermediate scission products and other molecular chains. By applying the 'resonance' concept and considering the energy of splitting of the chain molecule at the possible points, it is deduced that both the isoprene and dipentene are formed by fission at the ends of the rubber molecule, little if any of the latter arising from dimerization of pre-formed isoprene.

Details of another piece of fundamental research from the Association's laboratories were given by Dr. L. C. Bateman on the "Photochemical Breakdown 